CAMILA SOMBRA MUIÑOS DE ANDRADE

\title{
MOVIMENTOS MIGRATÓRIOS MISTOS E A CONDIÇÃO JURÍDICA DE REFUGIADO: uma relação tensional
}

Tese de Doutorado

Orientador: Professor Doutor GUILHERME Assis DE ALMEIDA

Faculdade de Direito da Universidade de São Paulo

São Paulo

2018 


\title{
MOVIMENTOS MIGRATÓRIOS MISTOS E A CONDIÇÃO JURÍDICA DE REFUGIADO: uma relação tensional
}

\begin{abstract}
Tese de Doutorado apresentada à Banca Examinadora do Programa de Pós-Graduação em Direito, da Faculdade de Direito da Universidade de São Paulo, como exigência parcial para obtenção do título de Doutora em Direito, na área de concentração em Direitos Humanos, sob orientação do Professor Doutor Guilherme Assis de Almeida.
\end{abstract}

Faculdade de Direito da Universidade de São Paulo

São Paulo 
Catalogação da Publicação

Serviço de Biblioteca e Documentação

Faculdade de Direito da Universidade de São Paulo

Andrade, Camila Sombra Muiños de

Movimentos Migratórios Mistos e a condição juridica de refugiado:

uma relação tensional / Camila Sombra Muiños de Andrade

orientador Guilherme Assis de Almeida -- São Paulo, 2018

136

Tese (Doutorado - Programa de Pós-Graduação em Direitos

Humanos) - Faculdade de Direito, Universidade de São Paulo, 2018

1. movimentos migratórios mistos. 2. refugiado. 3 . migrante 4 migrações forçadas. I. Almeida, Guilherme Assis de, orient. II. Título 
Nome: ANDRADE, Camila Sombra Muiños de.

Título: Movimentos migratórios mistos e a condição jurídica de refugiado: uma relação tensional

Tese de Doutorado apresentada à Banca Examinadora do Programa de Pós-Graduação em Direito, da Faculdade de Direito da Universidade de São Paulo, como exigência parcial para obtenção do título de Doutora em Direito, na área de concentração em Direitos Humanos, sob orientação do Professor Doutor Guilherme Assis de Almeida.

Data de aprovação:

Banca examinadora

Prof.Dr. Instituição:

Julgamento: Assinatura:

Prof.Dr. Instituição:

Julgamento: Assinatura:

Prof.Dr. Instituição:

Julgamento: Assinatura:

Prof.Dr. Instituição:

Julgamento: Assinatura:

Prof.Dr. Instituição: Julgamento: Assinatura:

Prof.Dr. Instituição: Assinatura: 
Para a minha familia. Em especial, aos meus pais e Diogo, por estarem sempre ao meu lado. 


\section{Agradecimentos}

Ao meu orientador, Professor Guilherme Assis DE AlmeIDA, pela confiança, disponibilidade e suporte em todo o período do doutorado. E, principalmente, pela sorte de um aprendizado que ultrapassa os limites deste trabalho.

À Professora Liliana Jubilut, por todas as sugestões de leituras e valiosas recomendações ao desenvolvimento deste trabalho.

À Professora MAÍRA MACHADO, por sua leitura atenta do texto na qualificação e sugestões que me ajudaram a revisar a estrutura da pesquisa.

À RIVANA RICARTE, por compartilhar comigo cada etapa do doutorado, desde o seu início.

À ERIKA Pires Ramos e à Thais Menezes, sempre disponíveis para trocar experiências sobre o processo de pesquisa.

À Maria BeAtriz NogueIRA, por suas sugestões de textos, livros, e, sobretudo, por seu importante auxílio para a organização do trabalho.

A Heike Drotbohm, Isabel Lima e João Carlos Jarochinski Silva, pelas sugestões de caminhos para esta pesquisa.

A Maya MANZi e DenISE KeY, pelo apoio na revisão das traduções.

Aos professores e funcionários da Faculdade de Direito da USP. 


\section{RESUMO}

ANDRADE, Camila Sombra Muiños de. Movimentos migratórios mistos e a condição jurídica de refugiado: uma relação tensional. 2018. 136f. Tese (Doutorado) - Faculdade de Direito, Universidade de São Paulo, São Paulo, 2018.

A Convenção de Genebra de 1951 estabeleceu direitos específicos para as pessoas refugiadas, como a não devolução a país onde possa estar em risco. Entretanto, não há um regime jurídico equivalente para as pessoas definidas como migrantes. Tradicionalmente, argumentou-se que, enquanto os primeiros haviam sido forçados ao deslocamento, os segundos haviam migrado por razões voluntárias. Estudos acadêmicos, contudo, passaram a destacar que fatores políticos, econômicos e ambientais estão associados enquanto causas das migrações, sejam estas em um contexto de maior ou menor grau de autonomia. Ao mesmo tempo, organizações internacionais, como o ACNUR, passaram a observar que refugiados e migrantes, em seu deslocamento, compartilhavam percursos, riscos e vulnerabilidades. A categoria de movimentos migratórios mistos é uma das expressões dessa dupla complexidade do deslocamento humano, em relação tanto aos seus motivos quanto às suas trajetórias. Mas como essa categoria relaciona-se à condição jurídica de refugiado? Essa é a pergunta que guia a presente investigação, dividida em duas partes. A primeira parte trata da elaboração dessa categoria. Nesse sentido, são analisadas as abordagens acadêmicas que desafiaram a divisão binária de migrações em forçadas e voluntárias, assim como as narrativas políticas sobre a relação entre migração e refúgio no período de 1950 ao final dos anos 1990. A segunda parte é dedicada ao desenvolvimento posterior da categoria, no período de 2001 a 2015. Nesse contexto, são investigadas as novas abordagens binárias ao deslocamento humano, as quais, centradas na perspectiva de segurança, dividem os movimentos migratórios em legais/ilegais. Percorridos esses sessenta e cinco anos de narrativas sobre a relação entre refúgio e migração, conclui-se: movimentos migratórios mistos e a condição jurídica de refugiado aproximam-se e afastam-se, em uma relação tensional.

Palavras-chave: 1. Movimentos migratórios mistos. 2. Refugiado. 3. Migrante. 4. Migrações forçadas. 


\begin{abstract}
ANDRADE, Camila Sombra Muiños de. Mixed Migratory Movements and refugee legal status: a tense relationship. 2018. 136pp. Thesis (Doctorate) - Faculty of Law, University of São Paulo (USP), São Paulo, 2018.

The 1951 Geneva Convention established specific rights for refugees, such as not returning them to a country where they may be at risk. However, there is no equivalent legal regime for people defined as migrants. Traditionally, it has been argued that while the former were forcibly displaced, the latter migrated voluntarily. Nevertheless, academic studies began to highlight that political, economic and environmental factors are associated as the reason for migrations, whether they are in a context of a greater or lesser degree of autonomy. At the same time, international organizations, such as the United Nations Refugee Agency (UNHCR), began to observe that refugees and migrants shared routes, risks and vulnerabilities when displaced. The mixed migratory movement category is one of the expressions of this dual complexity of human displacement, both in relation to reasons and trajectories. But how is this category related to refugee legal status? This question guides the investigation, which is divided into two sections. The first covers the creation of this category. Therefore, an analysis is carried out of the academic approaches which challenged the binary division of forced and voluntary migrations and the political narratives about the relationship between migration and refuge between 1950 and the end of the 1990s. The second section focuses on the later development of the category during the period 2001 to 2015. In this context, the new binary approaches to human displacement which, centered on the perspective of security, dividing migratory movements into legal/illegal, are investigated. Having covered sixtyfive years of narratives about the relation between refuge and migration, the following conclusion is reached: mixed migratory movements and refugee legal status come closer and distance themselves, in a tense relationship.
\end{abstract}

Keywords: 1 . Mixed migratory movements. 2. Refugee. 3. Migrant. 4. Forced migrations. 


\section{RÉSUMÉ}

ANDRADE, Camila Sombra Muiños de. Mouvements Migratoires Mixtes et la condition juridique du réfugié: une relation de tension. 2018. 136f. Thèse (Doctorat) - Faculté de Droit, Université de São Paulo, São Paulo, 2018.

La Convention de Genève de 1951 a établi des droits spécifiques pour les personnes réfugiées, tel que le principe du non-refoulement sur un pays où elles pourraient être à risque. Néanmoins, il n'y a pas un régime juridique équivalent pour les personnes définies comme migrants. Traditionnellement, il a été avancé que, tandis que les premiers ont été forcés au déplacement, les deuxièmes avaient migré pour raisons volontaires. Études académiques ont commencé cependant à souligner que des facteurs politiques, économiques et environnementaux sont associés en tant que causes des migrations, soit celles-ci dans un contexte de plus haut ou bas degré d'autonomie. En même temps, des organisations internationales comme l'UNHCR ont commencé à observer que réfugiés et migrants, lors de leurs déplacements, partageaient des parcours, risques et vulnérabilités. La catégorie des mouvements migratoires mixtes est l'une des expressions de cette double complexité du déplacement humain, tant qu'en relation à ses motifs, quant à ses trajectoires. Mais comment cette catégorie se relie-t-elle à la condition juridique du réfugié? Celle-ci est une question qui mène la présente recherche, divisée en deux parties. La première consiste en l'élaboration de cette catégorie. En ce sens, seront analysées les démarches académiques qui ont défié la division binaire de migrations en forcées et volontaires, ainsi que les narratives politiques sur la relation entre migration et refuge dans la période de 1950 jusqu'à la fin des années 1990. La deuxième partie est consacrée au développement postérieur de la catégorie, dans la période de 2001 à 2015. Dans ce contexte, sont recherchées les nouvelles démarches binaires au déplacement humain, lesquelles, centrées sur la perspective de sécurité, scindent les mouvements migratoires en légaux/illégaux. Écoulés ces soixante-cinq ans de narratives sur la relation entre refuge et migration, il est conclu que: mouvements migratoires mixtes et la condition juridique de réfugié s'approchent et s'écartent, dans une relation de tension.

MOTS-CLÉS: 1. Mouvements migratoires mixtes. 2. Réfugié. 3. Migrant. 4. Migrations forcées. 
"The political and bureaucratic dynamics of labelling constitute a constructive field of studies of itself, even as such studies tell more about the agents, structures, and effects of labelling than about the people labelled. [...]. One example is the politically significant notion of 'mixed flows', the 'complex' population movements of irregular migrants, refugees, asylum seekers, stateless persons, unaccompanied minors, and other vulnerable persons travelling in irregular manner along similar routes, using similar means of travel". 


\section{SUMÁRIO}

INTRODUÇÃO

Parte I - Movimentos migratórios mistos: emergência da categoria

1.1 Abordagens analíticas e a emergência da categoria de movimentos migratórios mistos

1.1.1 Abordagens de continuum

1.1.2 Abordagens de categorização 30

1.1.3 Abordagens metodológicas 33

1.2 Narrativas políticas e a emergência da categoria de movimentos migratórios mistos

$1.2 .1 \quad 1950$ a 1970 40

1.2.2 Anos 1980 45

1.2.3 Anos 1990 51

Parte II - Movimentos migratórios mistos: desenvolvimento do conceito 75

2.1 Abordagens analíticas e a transformação da categoria de movimentos migratórios mistos 78

2.1.1 Novas abordagens de categorização 78

2.1.2 Novas abordagens binárias. 83

2.1.3 Novas abordagens, uma mesma categoria 87

2.2 Narrativas políticas e a transformação do conceito de movimentos migratórios mistos 
CONCLUSÃO 


\section{INTRODUÇÃO}

A Al Jazeera “não falará em 'migrantes' do Mediterrâneo". Assim anunciou a rede de comunicação em agosto de 2015. A partir desse marco, as pessoas que atravessam o Mediterrâneo em direção à Europa, viajando em barcos vulneráveis e, muitas vezes, vítimas de tragédias no mar, passaram a ser referidas pela rede como "refugiadas" (MALONE, 2015).

Em justificativa a essa decisão, a Al Jazeera ressaltou que "a palavra migrante tornou-se um guarda-chuva amplo e impreciso para esta história complexa". ${ }^{1}$ Isso porque o termo "migrante", tal como empregado pela mídia, desumaniza as pessoas, em sua opinião. Além disso, acrescentou que a maior parte dessas pessoas advém de países marcados por situações de forte conflito, como Síria, Afeganistão, Iraque e Somália. Por conseguinte, argumentou que não podem ser consideradas migrantes econômicos, como pretendem discursos políticos de governos. Em conclusão, para a rede, "migrante é uma palavra que retira a voz das pessoas em sofrimento. Substituí-la por refugiados é - de um modo mínimo - uma tentativa de dar [essa voz] a alguns de volta" (MALONE, 2015). ${ }^{2}$

A decisão editorial, contudo, suscitou controvérsias. Poucos dias depois, a BBC, outra rede internacional de notícias, reagiu em defesa do uso da palavra "migrante" como expressão da variedade de pessoas em movimento. A rede afirmou a neutralidade do termo com base na definição encontrada no dicionário inglês de Oxford, em que migrante é "alguém que se move, temporária ou permanentemente, de um lugar, área ou país de residência para outro"3 ${ }^{3}$ RUZ, 2015). Também em embate direto, a acadêmica Judith Vonberg (2015) argumentou que a opção da Al Jazeera, em verdade, teria como efeito não a humanização dos refugiados, mas a falta de compaixão perante aqueles classificados como migrantes. Assim, em sua opinião, a rede estaria contribuindo para o discurso de fechamento de fronteiras dos países. E, acrescentou Vonberg, são tênues as diferenças

1 Tradução livre. Original em inglês: "The word migrant has become a largely inaccurate umbrella term for this complex story" (MALONE, 2015).

2 Tradução livre. Original em inglês: "Migrant is a word that strips suffering people of voice. Substituting refugee for it is - in the smallest way - an attempt to give some back" (MALONE, 2015).

3 Tradução livre. Original em inglês: "one who moves, either temporarily or permanently, from one place, area, or country of residence to another" (RUZ, 2015). 
entre refugiados e migrantes, sobretudo quando advêm de países marcados por situações de conflito somadas a graves crises socioeconômicas (VONBERG, 2015).

A discussão acalorada não é apenas teórica. A essencial distinção entre refugiados e migrantes fundamenta-se na ideia de que os primeiros "precisam migrar" enquanto os segundos "escolhem migrar" (CARLING, 2017). E da definição de uma pessoa como refugiada ou migrante derivam consequências, tais como o acesso a territórios, a garantia de não devolução a país onde sua vida e integridade possam ser postas em risco, além do direito à documentação, ainda que cruze as fronteiras de modo irregular. Com efeito, esses são alguns dos direitos fundamentais que compõem a proteção internacional das pessoas refugiadas, com amparo na Convenção das Nações Unidas de 1951 Relativa ao Estatuto dos Refugiados (Convenção de Genebra de 1951) e no respectivo Protocolo de 1967 Relativo ao Estatuto dos Refugiados (Protocolo de 1967). A mesma diferenciação reflete-se em políticas e legislações regionais e nacionais, que determinam respostas particulares a refugiados e migrantes em direitos sociais, econômicos e culturais (CARLING, 2017).

O fato é que essas pessoas "estão no mesmo barco, mas não na mesma realidade", resume Erik Christophersen (2016), do Norwegian Refugee Council. E, observa o autor, ao se olhar para um grupo de pessoas que chegam a um dado destino internacional, não é possível antecipar quem será reconhecido como refugiado. Nem mesmo o grau de vulnerabilidade em que se encontram é um indicativo confiável. Nessa realidade de direitos, o que importa é a conformidade aos parâmetros jurídicos internacionais (CHRISTOPHERSEN, 2016).

De acordo com esses instrumentos, o conceito de refugiado se refere à pessoa que, encontrando-se fora do seu país de residência, a este não pode ou deseja retornar em razão de fundado temor de perseguição por raça, religião, nacionalidade, grupo social ou opinião política (UN GENERAL ASSEMBLY, 1951, art. $1^{\circ}$ ). O conceito de migrante, 
contudo, não encontra similar delimitação pelo direito internacional, de forma que não há uma definição universalmente aceita sobre seu conteúdo (CARLING, 2015). ${ }^{4}$

Castles resume que "o ponto essencial aqui é que um regime legal internacional para proteger e assistir refugiados existe, ao passo em que este regime é largamente ausente tanto para outros tipos de migrantes forçados quanto para migrantes econômicos" (CASTLES, 2007, p. 28). ${ }^{5}$ Como resultado, a própria relação estabelecida entre os conceitos de refugiado e migrante é controversa. O debate, neste ponto, centra-se na seguinte questão: "São os refugiados uma subcategoria de migrantes?" (CARLING, 2015).

As perspectivas que Carling denomina "inclusivas" sustentam que sim, não distinguindo entre movimentos voluntários e forçados (2017). Nicolas Van Hear defende esse argumento em seu livro New diásporas: The mass exodus, dispersal and regrouping of migrant communities (VAN HEAR, 1998b). Observa, nesse sentido, que o conceito de migrante abrange a ampla variedade de pessoas em trânsito, sejam elas migrantes sem documentos, estudantes, trabalhadores, residentes temporários ou permanentes, estando inclusos refugiados e solicitantes de refúgio (VAN HEAR, 1998b, p. 41). Em apoio a sua posição, nota que não é incomum que uma pessoa passe de uma categoria migratória a outra (quando, por exemplo, se esgota o tempo da sua autorização de residência em determinado país) (VAN HEAR, 1998b, p. 41). Outro exemplo é a posição da Organização Internacional para as Migrações (OIM), que define a pessoa migrante como:

[...] qualquer pessoa que está se movendo ou se moveu cruzando uma fronteira internacional ou dentro de um Estado distante do seu local de residência habitual, independentemente: (1) do status jurídico da pessoa; (2) se o seu movimento é voluntário ou involuntário; (3) quais são as causas do seu movimento; (4) qual a duração da sua permanência (IOM, Key Migration Terms) ${ }^{6}$

4 A expressão específica "trabalhador migrante" encontra definição na Convenção da ONU sobre a Proteção de todos os trabalhadores migrantes e membros de suas famílias, que determina, em seu art. $2^{\circ}$ : “1. A expressão 'trabalhador migrante' designa a pessoa que vai exercer, exerce ou exerceu uma atividade remunerada num Estado de que não é nacional”. Ratificada por países de origem de trabalhadores migrantes, a Convenção não encontrou adesão naqueles de destino.

5 Tradução livre. Original em inglês: "The key point here is that an international legal regime to protect and assist refugees does exist, while such a regime is largely absent both for other types of forced migrant and for economic migrants" (CASTLES, 2007, p. 28).

6 Tradução livre. Original em inglês: "Migrant - IOM defines a migrant as any person who is moving or has moved across an international border or within a State away from his/her habitual place of residence, 
Em contraponto, "Refugiados não são migrantes", asseverou Erika Feller (2005), do Alto Comissariado das Nações Unidas para Refugiados (ACNUR). Para Feller, “é perigoso e prejudicial à proteção dos refugiados confundir os dois grupos, do ponto de vista terminológico ou outro" (FELLER, 2005, p. 27). ${ }^{7}$ Feller insurge-se contra a consideração dos refugiados enquanto subgrupo dos migrantes, pois, "na medida em que as divisões entre 'migrante' e 'refugiado' são borradas, assim também são as distinções entre controle migratório e proteção aos refugiados” (FELLER, 2005, p. 27). ${ }^{8}$ Como expressão desse posicionamento institucional, o ACNUR (2015) utiliza a terminologia "refugiados e migrantes" para referir-se a situações complexas, como os movimentos migratórios que cruzam o Mediterrâneo e outras regiões. Jorgen Carling (2017) denomina essa posição de "residualista", visto que migrantes são considerados pessoas que se deslocaram de seu local de origem, mas não são refugiados.

Mas quando os refugiados deixaram de ser migrantes? Essa pergunta guiou a pesquisa histórica de Katy Long relativa ao período de 1920 a 1950, antecedente, portanto, aos referidos instrumentos internacionais de proteção aos refugiados. Em seu estudo, Long revela como o conceito de refugiado, inicialmente compreendido como uma subcategoria de migrante, foi sendo interpretado aos poucos por meio de uma identidade autônoma e regime jurídico próprio (LONG, 2013, p. 4).

Não apenas a perseguição política, mas também a fome era considerada como causa integrante da identidade de refugiado no contexto da Revolução de 1917 e da guerra civil russa. Fridjof Nansen, nomeado pela Liga das Nações como Alto Comissário para Refugiados Russos, trabalhou para permitir a migração de refugiados a outros territórios onde pudessem ter autossuficiência econômica. O passaporte Nansen, documento de viagem para refugiados, chegou a ser reconhecido por mais de cinquenta países (LONG, 2013, p. 7-10). Também é reflexo do mesmo momento histórico o fato de que, entre 1925 e 1929, a Organização Internacional do Trabalho (OIT) foi a instituição que assumiu a

regardless of (1) the person's legal status; (2) whether the movement is voluntary or involuntary; (3) what the causes for the movement are; or (4) what the length of the stay is. IOM concerns itself with migrants and migration-related issues and, in agreement with relevant States, with migrants who are in need of international migration services" (IOM, Key Migration Terms).

7 Tradução livre. Original em inglês: "Secondly it is dangerous, and detrimental to refugee protection, to confuse the two groups, terminologically or otherwise" (FELLER, 2005, p.27).

8 Tradução livre. Original em inglês: "As the line between 'migrant' and 'refugee' blurs, so does the distinction between migration control and refugee protection" (FELLER, 2005, p.27). 
responsabilidade de encontrar soluções para os refugiados, ${ }^{9}$ conectando-os com oportunidades profissionais existentes para além do continente europeu (LONG, 2013, p. 7-10). ${ }^{10}$

No entanto, a crise dos anos 1930 impulsionou mudanças na abordagem ao tema do refúgio. $\mathrm{O}$ fechamento de fronteiras afetou tanto os migrantes econômicos como os refugiados, reduzindo as possibilidades de soluções pela via do trabalho. Nas palavras de Long, "foi o encerramento das vias de migração que condenou muitos refugiados, pois, quando essas se fecharam, foram negados aos refugiados não apenas o acesso a soluções, mas ao próprio refúgio" (LONG, 2013, p. 13). ${ }^{11}$ Como reflexo dessa nova conjuntura, a responsabilidade sobre o escritório do Alto Comissário deixou o âmbito da OIT e retornou à Liga das Nações. Nesse momento, as Convenções de Refugiados de $1933^{12}$ e $1938,{ }^{13}$ que já traziam o princípio de não devolução de pessoas refugiadas, tiveram pouca adesão entre os países (LONG, 2013, p. 11-13).

No contexto pós-Segunda Guerra Mundial, embora mantidos programas de mobilidade laboral, fortaleceu-se o argumento humanitário de que os refugiados deveriam ter acesso à proteção. Como observa a mesma autora, nesse momento, essas pessoas eram apresentadas pelos países ora como refugiadas, ora como trabalhadoras migrantes (LONG, 2013, p. 13-15). Exemplo disso é o programa "European Voluntary Workers", em que pessoas da Europa Central e do Leste deixavam campos de refugiados para trabalhar no Reino Unido por um período mínimo de 12 meses. Ao mesmo tempo em que o programa

9 Nesse período de 1925 a 1929, o escritório do Alto Comissário para Refugiados passou a estar vinculado, dos pontos de vista administrativo e financeiro, à OIT. Essa decisão decorreu justamente do fato de que refúgio e acesso ao trabalho eram temas relacionados de maneira muito próxima. Essa vinculação administrativa se manteve até 1929. Em 1930, o escritório do Alto Comissário voltou a situar-se no âmbito do Secretariado das Nações Unidas (Refugees Mixed Archival Group (Nansen Fonds). Unites Nations Archives, Geneva, 1947. Disponível em: <http://biblio-archive.unog.ch/detail.aspx?ID=256>. Acesso em: 4 set. 2017).

10 Long traz o exemplo de acordo entre a OIT e o Estado de São Paulo no Brasil, em 1926, para que o governo recebesse 265 refugiados a cada mês (LONG, 2013, p. 10).

11 Tradução livre. Original em inglês: "it was the closure of migration channels that damned many would-be refugees because as these closed they were denied access not only to a solution, but to asylum" (LONG, 2013, p. 13).

12 Convenção Relativa ao Status Internacional dos Refugiados, de 28 de outubro de 1933 (LEAGUE OF NATIONS, 1933).

13 Convenção Relativa ao Status de Refugiados Provenientes da Alemanha (LEAGUE OF NATIONS, 1938). 
foi apresentado à população local em termos humanitários, requisitos para a participação eram a boa situação de saúde e condição apta ao trabalho (LONG, 2013, p. 13-15).

Os anos 1950 foram marcados pela constituição do ACNUR e pela ratificação da Convenção de Genebra de 1951. Nesse período, o ACNUR buscou ampliar soluções para as pessoas mais vulneráveis, como os idosos e os doentes, por meio de apelos aos governos para que programas de reassentamento operassem a partir de critérios humanitários (LONG, 2013, p. 19-20). Igualmente, soluções econômicas para a situação dos refugiados continuaram sendo consideradas relevantes. A Convenção de Genebra de 1951, nesse sentido, faz referência explícita ao direito de trabalho e à necessidade de tratamento não discriminatório às pessoas refugiadas no âmbito laboral (LONG, 2013, p. 15-16). Com efeito, Long ressalta que

isso sugere que aqueles que redigiram a Convenção de Genebra de 1951 esperavam não apenas que refugiados continuassem a mover-se como migrantes, mas que pela virtude da contribuição desses refugiadosmigrantes como trabalhadores, também teriam acesso a caminhos acelerados de integração (LONG, 2013, p. 16). ${ }^{14}$

No entanto, à medida que os procedimentos de admissão dos países passaram a se atrelar ao fundamento humanitário, e não à ideia de recrutamento laboral, as identidades de refugiado e migrante foram sendo estabelecidas enquanto categorias distintas (LONG, 2013, p. 21).

A pesquisa de Long evidencia, assim, que, a depender do momento histórico, as identidades de refugiado e migrante foram apresentadas em maior ou menor grau de vinculação. Com efeito, quando as oportunidades laborais se apresentaram, refugiados ingressaram em programas de trabalho, ao passo que o argumento centrado no humanitarismo se revelou necessário, em especial, para a busca de soluções aos mais vulneráveis e nos períodos de recessão econômica. Não houve, observa a mesma autora, um momento determinado em que essas identidades foram definitivamente separadas (LONG, 2013, p. 21).

14 Tradução livre. Original em inglês: "This suggests not only that those drafting the 1951 Convention expected refugees to continue to move as migrants, but that by virtue of these refugee-migrants' contribution as labourers, they should be able to access an accelerated pathway to integration" (LONG, 2013, p. 16). 
Independentemente do posicionamento adotado, se inclusivo ou residualista, a distinção tradicional entre movimentos voluntários e forçados vem sendo desafiada pela categoria de movimentos migratórios mistos. Nas palavras de Khalid Koser e Susan Martin:

Este termo tem significados diferentes para diferentes instituições e em diferentes contextos, mas geralmente é usado para descrever uma situação em que os migrantes se movendo por motivações amplamente diferentes são difíceis de distinguir pois eles se movem entre os mesmos países de origem, trânsito e destino, muitas vezes com a assistência do mesmo contrabandista ou agente migratório, e muitas vezes no mesmo avião, barco ou caminhão. O exemplo mais citado são os barcos que atravessam o Mediterrâneo desde a África subsaariana e do norte até o sul da Europa. O desafio para os formuladores de políticos é distinguir aqueles que têm direito à assistência e proteção internacionais, daqueles que não o tem (KOSER; MARTIN, 2011, p. 4-5). ${ }^{15}$

Essa definição dos movimentos migratórios atuais pressupõe uma dimensão multicausal do deslocamento humano. Ao mesmo tempo, determina o desenvolvimento de uma nova abordagem estratégica das organizações multilaterais com vistas a essa realidade, no sentido de incluir o tema da proteção aos refugiados no contexto mais amplo de migração internacional. Trata-se, assim, de uma categoria com duas dimensões: uma analítica e outra política, conforme destacado por Van Hear (2011). É o estudo da relação entre essas dimensões da categoria de movimentos migratórios mistos e a condição jurídica de refugiado que circunscreve a presente investigação.

Para tanto, será feito um estudo das abordagens analíticas e narrativas políticas relacionadas à categoria de movimentos migratórios mistos. O trabalho se divide em duas partes, tendo como referência o tempo das discussões da categoria no âmbito das organizações multilaterais. Na primeira, será investigada a emergência da categoria, considerando o período de 1951 a 2000, e, na segunda, será observada a sua trajetória

15 Tradução livre. Original em inglês: "This term has different meanings for different instititutions and in different settings, but it is most usually used to describe a situation where migrants moving for broadly different motivations are difficult to discern because they move between the same origin, transit and destination countries, often with the assistance of the same smuggler or migration agent, and often in the same airplane, boat or truck. The most often-cited example is the boats that traverse the Mediterranean from sub-Saharan and North Africa to Southern Europe. The challenge for policy makers is to distinguish those who are entlitled to international assistance and protection, from those who are not" (KOSER, MARTIN, 2011, p. 4-5). 
posterior, no período compreendido entre 2001 e 2015. Permeando essa análise estão os principais estudos acadêmicos que orientam o tema.

A escolha deste percurso se deve ao fato de que "nenhum conjunto de instituições jurídicas ou normas existem em separado das narrativas que o situam e lhe proporcionam significado", conforme observou Robert Cover no texto "Nomos and Narrative" (1983, p.4). Por meio desta trajetória, como sugerido por Cover, "[...] o direito se torna não meramente um sistema de regras a serem observadas, mas o mundo no qual nós habitamos" (COVER, 1983, p. 5). 


\section{CONCLUSÃO}

No final de 2015, o tema da "crise migratória" na Europa voltou-se para a Grécia. Esse país passou a ocupar o protagonismo que era então da Itália no recebimento de pessoas deslocadas via o Mediterrâneo, principalmente a partir da Líbia. Apenas três países de origem - Síria, Afeganistão e Iraque - representavam cerca de 90\% das pessoas que chegavam à Grécia naquele momento. Países esses notadamente marcados por situações de grave violência e perseguição (CRAWLEY; SKLEPARIS, 2018, p. 48).

Apesar de a relação de nacionalidades indicar preocupações de proteção internacional, líderes de países como Hungria e Eslováquia declararam categoricamente: essas pessoas não são refugiadas, mas migrantes em busca de melhores condições de vida (CRAWLEY; SKLEPARIS, 2018, p. 48).

Adicione-se, então, mais um dado a esse contexto. Muitas dessas pessoas que chegavam à Europa não vinham diretamente de seus países de origem. Por meses (ou mesmo anos), antes de chegarem ao continente, viveram em outras localidades que não a sua de origem, contrariando a ideia de que, no contexto de uma crise, é estabelecido um fluxo linear de pessoas até o seu destino final (CRAWLEY; SKLEPARIS, 2018, p. 49).

O seu percurso até a Europa deve ser considerado como uma continuação da fuga das situações de graves violações de direitos humanos, ou aquele que, inicialmente, era um refugiado, torna-se um migrante econômico?

O conceito de movimentos migratórios mistos oferece respostas distintas a essa pergunta.

A primeira permite considerar as múltiplas motivações que compõem o ato de migrar. Na perspectiva de um continuum de coerções e possibilidades, conforme proposto por Richmond e Van Hear, razões econômicas somam-se - e não invalidam - às razões de ordem política ou de perseguição que são preponderantes para o deslocamento. Situados no eixo de maior nível de coerção ao seu movimento, afirma-se, refugiados não são "apenas" migrantes, de forma que se justifica uma proteção específica do direito internacional, que considere suas vulnerabilidades e necessidades. Contudo, essa interpretação avança, 
passando a dispor que refugiados são também migrantes, trazendo consigo aspirações voltadas ao futuro e vínculos com olhar para o passado, formando redes de comunidades transnacionais também inseridas nos processos de globalização.

A segunda resposta propõe a separação daquele que se considera um grupo pequeno de pessoas com necessidades de proteção internacional de uma maioria de pessoas migrantes que se deslocam - muitas vezes sem documentos - em busca de oportunidades econômicas. Nessa leitura, trajetos e riscos são compartilhados entre os diversos grupos e perfis de pessoas migrantes, mas não suas motivações. Conforme essa perspectiva, quanto mais duradouro um conflito, mais difícil o encontro de "refugiados genuínos", como destacam Crawley e Skleparis (2018, p. 55). Assim, com o passar dos anos, realidades que eram consideradas como de refúgio passam a ser interpretadas como expressão dessa nova categoria, os "movimentos migratórios mistos".

Essas interpretações não são excludentes, conforme observado nos posicionamentos de acadêmicos, governos e organizações humanitárias.

Revelam, contudo, que os movimentos migratórios mistos e a condição jurídica de refugiado aproximam-se e contrapõem-se em uma relação tensional. 


\section{REFERÊNCIAS BIBLIOGRÁFICAS}

ALMEIDA, Guilherme Assis de. A proteção da pessoa humana no direito internacional: conflitos armados, refugiados e discriminação racial. São Paulo: Editora CLA, 2018.

BAKEWELL, Oliver. Research beyond the Categories: The Importance of Policy Irrelevant Research into Forced Migration. Journal of Refugee Studies, v. 21, n. 4, p. 432-453, 2008.

BETTS, Alexander. Comprehensive Plans of Action:iLepi:Insights from CIREFCA and the Indochinese CPA. New issues in refugee research. Working Paper No. 120, UNHCR, January 2006.

; DURIEUX, Jean François. Convention Plus as a Norm-Setting Exercise. Journal of Refugee Studies, v. 20, n. 3, p. 509-535, 2007.

; LOESCHER, Gil.; MILNER, James. UNHCR: The Politics and Practice of Refugee Protection into the 21st Century. New York: Routledge, 2008.

CARLING, Jorgen. Refugee Advocacy and the Meaning of "Migrants". Peace Research Institute Oslo. PRIO Policy Brief, fev. 2017. Disponível em: $<$ https://www.prio.org/utility/DownloadFile.ashx?id=326\&type=publicationfile $>$. Acesso em: 7 jul. 2017.

. Refugees are Also Migrants. And All Migrants Matter. Border Criminologies, University of Oxford, 2015. Disponível em: <http://bordercriminologies.law.ox.ac.uk/ refugees-are-also-migrants/>. Acesso em: 19 jun. 2016.

CASTLES, Stephen. The Migration-Asylum Nexus and Regional Approaches. In: KEEBONE, Susan; RAWLINGS-SANAEI, Felicity. New Regionalism and Asylum Seekers: Challenges Ahead. Berghan Books, 2007.

. Towards a Sociology of Forced Migration and Social Transformation. Sociology, v. 37, n. 1, p. 13-34, 2003. 
CHRISTOPHERSEN, Erik. In the same boat, but not in reality. Norwegian Refugee Council, 28 jul. 2016. Disponível em: <https://www.nrc.no/news/2016/july/in-thesame-boat-but-not-in-reality/>. Acesso em: 4 jul. 2017.

CIS CONFERENCE. Regional Conference to Address the Problems of Refugees, Displaced Persons, Other Forms of Involuntary Displacement and Returnees in the Countries of the Commonwealth of Independent States and Relevant Neighbouring States.Geneva, 30-31 May 1996.

COVER, Robert M. The Supreme Court, 1982 Term - Foreword: Nomos and Narrative. Faculty Scholarship Series, Paper 2705, 1983.

CRAWLEY, Heaven; SKLEPARIS, Dimitris. Refugees, migrants, neither, both: categorical fetishism and the politics of bounding in Europe's "migration crisis". Journal of Ethnic and Migration Studies, v. 44, n. 1, p. 48-64, 2018.

CRISP, Jeff. Beyond the nexus: UNHCR's evolving perspective on refugee protection and international migration. New Issues in Refugee Research. UNHCR Research paper $\mathrm{n}^{\circ}$ $155,2008$.

- Refugees, Persons of Concern, and People on the Move:itipe Broadening Boundaries of UNHCR. Refuge, v. 26, n. 1, 2009.

DERDERIAN, Katharine; SCHOCKAERT, Liesbeth. Respostas a fluxos migratórios mistos: uma perspectiva humanitária. Sur, Rev. Int. Direitos Human. [on-line], v. 6, n. 10, p. 116-119, 2009.

ELLIOT, Sarah. Refugee Protection in Mixed Migration - a UNHCR perspective pre and post summit. Refugee Law Initiative, Podcasts, 1 December 2016. Disponível em: $<$ https://rli.sas.ac.uk/resources/podcasts>. Acesso em: 30 nov. 2017.

ExCom. The Problem of Manifestly Unfounded or Abusive Applications for Refugee Status or Asylum. Executive Committee 34th session. No. 30 (XXXIV). Contained in United Nations General Assembly Document No. 12A (A/38/12/Add.1). 1983. 
FELLER, Erika. Preface. In: FELLER, Erika; TURK, Volker; NICHOLSON, Frances. Refugee Protection in International Law. UNHCR's Global Consultations on International Protection. Cambridge University Press, 2003.

. Refugees are not Migrants. Refugee Survey Quarterly, v. 24, issue 4, p. 27-35, 2005.

. Revitalizing the Refugee Protection Regime: The Road Ahead as the 1951

Convention turns 50. Statement to the 51st Session of ExCom, Geneva, 3 October 2000 .

FIDDIAN-QASMIYEH, Elena; LOESCHER, Gil; LONG, Katy; SIGONA, Nando. Introduction: Refugee and Forced Migration Studies in Transition. The Oxford Handbook of Refugee \& Forced Migration Studies. Oxford University Press, 2014.

GEIGER, Martin; PÉCOUD, Antoine. The Politics of International Migration Management. Palgrave Macmillan, 2010.

GOEDHART, Gerrit Jan van Heuven. Remarks to the 470th Meeting of the Third Committee of the United Nations General Assembly. United Nations High Commissioner for Refugees. Geneva, 9 December 1952.

. Speech delivered to the Conference on Migration. Brussels, 1951.

GUTERRES, Antonio. Closing Statement at the Fifty-sixth Session of the Executive Committee of the High Commissioner's Programme (ExCom), Geneva, 7 October $2005 b$.

- Millions Uprooted: Saving Refugees and the Displaced. Foreign Affairs, September-October 2008. Disponível em: <https:/www.foreignaffairs.com/ articles/2008-09-01/millions-uprooted>. Acesso em: 2 abr. 2018.

Opening Statement at the Fifty-sixth Session of the Executive Committee of the High Commissioner's Programme (ExCom), Geneva, 3 October 2005a. 
- Opening Statement at the High Commissioner's Dialogue on Protection Challenges, Geneva, 11 December $2007 b$.

. Statement to the Third Committee of the United Nations General Assembly, 62nd Session, New York, 8 November 2007a.

HAMMERSTAD, Anne. The Securitization of Forced Migration. In: FIDDIANQASMIYEH, Elena; LOESCHER, Gil; LONG, Katy; SIGONA, Nando. The Oxford Handbook of Refugee \& Forced Migration Studies. Oxford University Press, 2014.

HARTLING, Paul. Statement to the Executive Committee of the High Commissioner's Programme. Thirty-third session. Geneva, 11 October 1982.

HOCKÉ, Jean-Pierre. Statement to the Third Committee of the United Nations General Assembly, 7 November 1986.

- Statement at the Seminar on International Humanitarian Law in the Contemporary World, Moscow, 4-6 June 1987.

INTERNATIONAL LABOUR ORGANIZATION (ILO). The asylum-migration nexus: refugee protection and migration perspectives from ILO. Global Consultations on International Protection. $2^{\text {nd }}$ Meeting. International Migration Branch, 28 June 2001.

INTERNATIONAL ORGANIZATION FOR MIGRATION (IOM). IOM History: 1950s. Disponível em: <https://www.iom.int/1950s>. Acesso em: 30 jul. 2017. - Key Migration Terms. Disponível em: <https://www.iom.int/key-migrationterms>. Acesso em: 5 jul. 2017.

JUBILUT, Liliana Lyra. O direito internacional dos refugiados e sua aplicação no ordenamento jurídico brasileiro. São Paulo: Método, 2007.

KHAN, Prince Sadruddin Aga. Lecture on legal problems relating to refugees and displaced persons. The Hague Academy of International Law, 4-6 August 1976. 
. Opening Statement to the Executive Committee of the High Commissioner's Programme. Twentieth session, 20 October 1969.

KOSER, K.; MARTIN, S. (Eds.). The Migration-Displacement Nexus: Patterns, Processes and Policies. Oxford: Berghan Books, 2011.

KUMIN, Judith. Europe: the debate over asylum - an uncertain direction. Refugees Magazine, issue 113, 1999.

KUNZ, E. F. The Refugee in Flight: Kinetic Models and Forms of Displacemen. The International Migration Review, v. 7, n. 2, p. 125-146, 1973.

LEAGUE OF NATIONS. Convention concerning the Status of Refugees Coming From Germany. League of Nations Treaty Series, v. CXCII, n. 4461, 10 February 1938.

. Convention Relating to the International Status of Refugees. League of Nations, Treaty Series, v. CLIX, n. 3663, 28 October 1933.

LINDE, Thomas. Mixed Migration: A Humanitarian Counterpoint. Refugee Survey Quarterly, v. 30, n. 1, 2011.

LINDT, Auguste. R. Statement to the Third Committee of the United Nations General Assembly. United Nations High Commissioner for Refugees, 2 November 1959.

LOESCHER, Gil. The UNHCR and World Politics: A Perilous Path. Oxford: Oxford University Press (Kindle), 2001.

. UNHCR and Forced Migration. In: FIDDIAN-QASMIYEH, Elena; LOESCHER, Gil; LONG, Katy; SIGONA, Nando. The Oxford Handbook of Refugee \& Forced Migration Studies. Oxford University Press, 2014.

. UNHCR's Origins and Early History: Agency, Influence, and Power in Global Refugee Policy. Refuge, v. 33, 2017. 
LONG, Katy. When refugees stopped being migrants: Movement, labour and humanitarian protection. Migration Studies, v. 1, issue 1, p. 4-26, 13 Feb 2013.

LUBBERS, Ruud. Closing Statement at the Fifty-fith Session of the Executive Committee of the High Commissioner's Programme (ExCom), Geneva, 8 October 2004.

. Opening Statement at the Fifty-second Session of the Executive Committee of the High Commissioner's Programme (ExCom), Geneva, 1 October 2001.

. Opening Statement at the Fifty-third Session of the Executive Committee of the High Commissioner's Programme (ExCom), Geneva, 30 September 2002.

MALONE, Barry. Why Al Jazeera will not say Mediterranean "migrants". The word migrant has become a largely inaccurate umbrella term for this complex story. $A l$ Jaazera, 20 August 2015. Disponível em: <http://www.aljazeera.com/blogs/editorsblog/2015/08/al-jazeera-mediterranean-migrants-150820082226309.html>. Acesso em: 2 jul. 2017.

OGATA, Sadako. Closing Statement at the Fiftieth Session of the Executive Committee of the High Commissioner's Programme (ExCom), Geneva, 8 October 1999.

. Closing Statement at the Fifty-first Session of the Executive Committee of the High Commissioner's Programme (ExCom), Geneva, 6 October $2000 \mathrm{~b}$.

- Fortress Europe? Refugees and migrants: their human rights and dignity. Statement at the Akademie Graz International Conference, Graz, Austria, 23 May 1992.

. Mixed Migration: Strategy for Refugees and Economic Migrants. Harvard International Review, v. 17, n. 2, p. 30-33, Spring 1995.

Opening Statement at the Forty-seventh Session of the Executive Committee of the High Commissioner's Programme (ExCom), 7 October 1996. 
Opening Statement at the Forty-eighth Session of the Executive Committee of the High Commissioner's Programme (ExCom), 13 October 1997.

. Opening Statement at the Forty-ninth Session of the Executive Committee of the High Commissioner's Programme (ExCom), Geneva, Monday, 5 October 1998.

. Opening Statementat the Fifty-first Session of the Executive Committee of the High Commissioner's Programme (ExCom), Geneva, 2 October 2000a.

OGATA, Sadako; SEN, Amartya. "Human Security Now". Comission on Human Security. New York, 2003.

PARIS APPEAL. Refugees at the French National Assembly. Global Consultations, 16 June 2001.

REID, Sue. The tragic but brutal truth: They are not REAL refugees! Despite drowning tragedy thousands of economic migrants are still trying to reach Europe. Daily Mail, 28 maio 2016. Disponível em: <http://www.dailymail.co.uk/news/article3613603/The-tragic-brutal-truth-not-REAL-refugees-Despite-drowning-tragedythousands-economic-migrants-trying-reach-Europe.html>. Acesso em: 20 mar. 2018.

RICHMOND, Anthony. Global Apartheid. Refugees, Racism and the New World Order. Oxford University Press, 1994.

- Sociological Theories of International Migration: The Case of Refugees's. Current Sociology, v. 36, n. 7, p. 7-25, 1988.

RUZ, Camila. The battle over the words used to describe migrants. BBC News Magazine, 28 August 2015. Disponível em: <http://www.bbc.com/news/magazine-34061097>. Acesso em: 20 mar. 2018.

SCHEEL, Stephan; SQUIRE, Vicki. Forced Migrants as 'Illegal' Migrants. In: FIDDIANQASMIYEH, Elena; LOESCHER, Gil; LONG, Katy; SIGONA, Nando. The Oxford Handbook of Refugee \& Forced Migration Studies. Oxford University Press, 2014. 
SCHNYDER, Felix. Statement to the Third Committee of the United Nations General Assembly, 21 November 1963.

. Statement to the Third Committee of the United Nations General Assembly at its twentieth session, 1 November 1965.

STEPPUTAT, Fin; SORENSEN, Ninna Nyberg. Sociology and Forced Migration. In: FIDDIAN-QASMIYEH, Elena; LOESCHER, Gil; LONG, Katy; SIGONA, Nando. The Oxford Handbook of Refugee \& Forced Migration Studies. Oxford University Press, 2014.

STOLTENBERG, Thorvald. Statement to the Extraordinary Session of the Executive Committee of the High Commissioner's Programme (ExCom), Geneva, 28 May 1990a.

. Opening Statement to the Executive Committee of the High Commissioner's Programme, Forty-first session, Geneva, 1 October 1990 b.

THOMAS, Manisha. UNHCR Candidates Talk Back. Mr. Antonio Guterres. The Newsletter of the International Council of Voluntary Agencies (ICVA). Special issue, part 2: Volume 7-2a, 14 April 2005.

TURK, Volker. Current Migration Flows: the task of protection. Refugee Survey Quarterly, v. 20, n. 2, 2001.

; RIERA, Jose; ENGLBRECHT, Walpurga. Overview of the Global Consultations on International Protection. Refugee Survey Quarterly, v. 22, n. 2/3, 2003.

TURTON, David. Conceptualising Forced Migration. Queen Elizabeth House International Development Centre University of Oxford. Working Paper Series. Working Paper $\mathrm{n}$. 12. October 2003a.

. Refugees and 'Other Forced Migrants'. Queen Elizabeth House International Development Centre University of Oxford. Working Paper Series. Working Paper n. 13. October 2003b. 
UN GENERAL ASSEMBLY. Agenda for Protection. Doc A/AC.96/965. Executive Committee of the High Commissioner's Programme. 26 June 2002

. Comprehensive consideration of the problems of refugees, returnees, displaced persons and related migratory movements. Doc. n. 50/151. Resolution adopted by the General Assembly on the Report of the Third Commitee A/50/632. Fiftieth Session, 21 December 1995.

. Convention Relating to the Status of Refugees, United Nations, Treaty Series, v. 189, 28 July 1951.

. Declaration and Comprehensive Plan of Action (CPA). Office of the United Nations High Comissioner for Refugees: International Conference on Indo-Chinese Refugees. Document A/44/523, 22 September 1989a.

. Implementing actions proposed by the United Nations High Commissioner for Refugees to strengthen the capacity of his Office to carry out its mandate. Res 58/153, 22 December 2003b.

Report by the High Comissioner to the General Assembly on Strenghthening the capacity of the office of the High Commissioner for Refugees to carry out its mandate. Doc. A/AC.96/980. Fifty-four Session, 20 August 2003a.

. Report of the United Nations High Commissioner for Refugees Report of the United Nations High Commissioner for Refugees. Doc A/4771/Rev.1, 1 January 1962.

. Report of the United Nations High Commissioner for Refugeesisêpisupplement n. 12 (A/34/12). 11 October 1979.

. Report of the United Nations High Commissioner for Refugees A/38/12, 17 August 1983.

Report of the United Nations High Commissioner for Refugees A/41/12, 1 August 1986. 
Report of the United Nations High Commissioner for Refugeesisepisupplement No.

12 (A/34/12). Thirty-fourth Session, 11 October 1989b.

. Statute of the office of the UNHCR. Resolution n. 428 (v), 14 December 1950.

UN NEWS. Lubbers resigns as UN refugee chief; Annan says move is in best interest of UN agency. 20 February 2005.

UNHCR. Ahmed's story. Necessary Risk. Telling the real story, 2016.

. Asylum Seekers. Who we help. Disponível em: <http://www.unhcr.org/asylumseekers.html>. Acesso em: 30 mar. 2018.

. Background note on the protection of asylum-seekers and refugees rescued at sea. Geneva. Global Consultations, 18 March 2002b.

. Declaration of State Parties to the 1951 Convention and or its 1967 Protocol Relating to the Status of Refuges. Ministerial Meeting of State Parties to the 1951 Convention and/or its 1967 Protocol relating to the Status of Refugees. Global Consultations. Geneva, 12-13 December 2001k.

. Desperate Journeys. Refugees and migrants entering and crossing Europe via the Mediterranean and Western Balkans routes. Bureau for Europe, February 2017a.

. Displacement in the CIS: the CIS Conference on Refugees and Migrants. Geneva: 1 May 1996.

- Executive Committee. Disponível em: <http://www.unhcr.org/executivecommittee.html>. Acesso em: 30 mar. 2018.

. Executive Committee of the High Commissionner's Program. Update on Global Consultations on International Protection 30 May 2001. Doc. EC/51/SC/CRP.12. Refugee Survey Quartely, v. 20, n. 4, 30 May 2001c. 
. Executive Committee of the High Commissionner's Program. Report of the Second Meeting in the Third Track. Doc A/AC.96/961. Global Consultations, Geneva, 28-29 June 2001d.

. Framework for Durable Solutions for Refugees and Persons of Concern. Core Group on Durable Solutions. Geneva, May 2003.

- Home. Previous High Commissioners. Sadako Ogata (Japan): 1991-2000. Disponível em: <http://www.unhcr.org/sadako-ogata-japan-1991-2000.html>. Acesso em: 10 abr. 2018.

. Home. Previous High Commissioners. Thorvald Stoltenberg (Norway): 19901990. Disponível em: <http://www.unhcr.org/thorvald-stoltenberg-norway-19901990.html>. Acesso em: 10 abr. 2018.

- NGO Background paper on the refugee and migration interface. Global Consultations, Geneva, 28-29 June 2001e.

. Progress Report: Convention Plus. FORUM/2005/6. Geneva, 8 November 2005.

. Protection of Refugees in the Context of Individual Asylum Systems. Chairman's Summary. Global Consultations. Geneva, 27-28 September 2001g.

. Reconciling Migration Control and Refugee Protection in the European Union: a UNHCR perspective. Office of the United Nations High Commissioner for Refugees. Geneva, October 2000b.

. Refugee Protection and Mixed Migration: A 10-Point Plan of Action. Geneva: UNHCR, January 2007c.

. 'Refugees' and 'Migrants' - Frequently Asked Questions (FAQs). 16 March 2016. Disponível em: <http://www.unhcr.org/afr/news/latest/2016/3/56e95c676/refugeesmigrants-frequently-asked-questions-faqs.html>. Acesso em: 5 jul. $2017 \mathrm{~b}$. 
Report of the Executive Committee of the Programme of the United Nations High Commissioner for Refugees Fiftieth Session, 4-8 October 1999.

- Selected Reference Materials Rescue at Sea. Maritime Interception and Stowaways. Geneva, November 2006 b.

. Summary Report of the first day. Ministerial Meeting of State Parties to the 1951 Convention and/or its 1967 Protocol relating to the Status of Refugees. Global Consultations. Geneva, 12 December 2001h.

. Summary Report of the second day. Ministerial Meeting of State Parties to the 1951 Convention and/or its 1967 Protocol relating to the Status of Refugees. Global Consultations. Geneva, 13 December 2001 i.

. The High-Level dialogue on International Migration and Development: UNHCR'S observations and recommendations. 28 June 2006a. Geneva: UNHCR.

- The Refugee Perspective. Recommendations on asylum processes, local integration and voluntary repatriation - made by refugees. Global Consultations. Rouen (France): 14-16 September 2001f.

. The State of the World's Refugees 1993: The Challenge of Protection. Geneva: UNHCR, 1993.

- The State of the World's Refugees 1995: In Search of Solutions. Geneva: UNHCR, 1995.

- The State of the World's Refugees 1997: A Humanitarian Agenda. Geneva: UNHCR, 1997.

. The State of the World's Refugees 2000: Fifty Years of Humanitarian Action. Geneva: UNHCR, 01 January 2000a.

. UNHCR, Refugee Protection and International Migration. UNHCR Geneva, 17 January 2007 a. 
. UNHCR'S Activities in relation to the asylum-migration nexus. EC/58/SC/CRP.12. Standing Committee 39th Meeting, 4 June 2007b.

. Update 31 January 2001. Global Consultations. Geneva, 31 Jan. 2001a.

. Update 15 May 2001. Global Consultations. Geneva, 15 May. 2001 b.

. Update January 2002. Global Consultations. Geneva, 2002a.

. Upholding Refugee Protection in the Face of Contemporary Challenges involving Mixed Flows. Ministerial Meeting of State Parties to the 1951 Convention and/or its 1967 Protocol relating to the Status of Refugees. Chairperson's Report on Roundtable 3. Global Consultations First Track. 13 December 2001j.

; IMPACT; ALTAI CONSULTING. Mixed Migration Trends in Lybia: Changing Dynamics and Protection Challenges. Evolutions of the journey and situations of refugees and migrants in Southern Lybia. 2017.

; IOM. Memorandum of Understanding between the United Nations High Commissioner for Refugees and the International Organization for Migration. Geneva, 15 May 1997.

; __ Refugee Protection and Migration Control: Perspectives from UNHCR and IOM. Doc. EC/GC/01/11. Global Consultations, 2nd meeting, 31 May 2001.

VAN HEAR, Nicholas. Editorial Introduction. Refugee Studies Programme, University of Oxford. Journal of Refugee Studies, v. 11, n. 4, 1998a.

. Mixed Migration. In: ANDERSON, B; KEITH, M. Migration: A COMPAS Anthology. Oxford, 2014.

. Mixed Migration: Policy Challenges. The Migration Observatory, 2011. Disponível em: <http://www.migrationobservatory.ox.ac.uk/policy-primers/mixedmigration-policy-challenges>. Acesso em: 5 jun. 2016. 
. New Diasporas: The Mass Exodus, Dispersal and Regrouping of Migrant Communities. London: Routledge/University College London Press, 1998 b.

; BRUBAKER, Rebecca; BESSA, Thais. Managing Mobility for Human Development: The Growing Salience of Mixed Migration. United Nations Development Programme Research Paper. Abril de 2009. Disponível em: $<$ https://mpra.ub.uni-muenchen.de/19202/1/MPRA_paper_19202.pdf $>$. Acesso em: 15 ago. 2017.

VONBERG, Judith. Al Jazeera will not say 'migrants' but we should. Huffpost United Kingdom, 25 de agosto de 2015. Disponível em: $<$ http://www.huffingtonpost.co.uk/judith-vonberg/al-jazeeramigrants_b_8036950.html>. Acesso em: 2 jul. 2017.

WHEN the war was over: European refugees after 1945. Briefing Paper 7. World Refugee Year, 1959-60, The University of Nothingham, 2012.

ZETTER, Roger. Labelling Refugees: Forming and Transforming a Bureaucratic Identity. Journal of Refugee Studies, v. 4, n. 1, p. 39-62, 1991.

. More Labels, Fewer Refugees: Making and Remaking the Refugee Label in an Era of Globalisation. Journal of Refugee Studies, v. 20, n. 2, p. 172-192, 2007.

. Protecting Forced Migrants: A State of the Art Report of Concepts, Challenges and Ways Forward. Federal Comission on Migration, 2014. 